\title{
Observation based gridded annual runoff estimates over Victoria, Australia
}

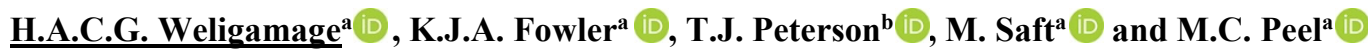 \\ ${ }^{a}$ Department of Infrastructure Engineering, University of Melbourne, Australia, \\ ${ }^{b}$ Department of Civil Engineering, Monash University, Australia \\ Email: hgardiyaweli@student.unimelb.edu.au
}

\begin{abstract}
In general, runoff generation is a highly variable process spatially. However, lack of spatially continuous runoff observations is a significant problem in surface hydrology due to gaps between gauged catchments and/or the need for within-catchment or ungauged catchment estimates. Recent efforts have produced programs for prediction in ungauged catchments, both in Australia (eg: Australian Water Resource Assessment - Landscape model $(A W R A-L))$ and internationally (eg: Prediction in Ungauged Basins $(P U B)$ ). While modelling approaches have many benefits (e.g. forecasting and projection), historic studies have the option of using observed data more directly. Adoption of geostatistical interpolation techniques potentially provides a more accurate method for generating spatially continuous runoff fields than modelling approaches. However, the use of geostatistical interpolation in hydrology is relatively uncommon because of the complex nature of hydrological connectivity. Here, we extend and apply top-kriging to spatially interpolate stream gauge data to produce gridded annual runoff across most of Victoria, Australia, from 1982-2012. Specifically, we extend the R-package rtop to allow kriging with external drift through the inclusion of spatial rainfall grids as a predictor. As expected, the resultant interpolated scheme provides a near exact match with observed values and acceptable performances in leave-one-out validation ( $\mathrm{R}^{2}$ of approximately 0.6 to 0.7 depending on year). The annual maps appropriately capture the variation in streamflow over time. Overall, this study illustrates that kriging can inform spatial runoff patterns and that the approach requires fewer inputs and less computational load than a complex numerical model. The methodology can be applied to any region with relatively minor adjustments. The dataset is open access in Zenodo repository at https://doi.org/10.5281/zenodo.5454798.
\end{abstract}

Keywords: Gridded runoff, top-kriging, rtop package, prediction in ungauged basins 


\section{INTRODUCTION}

Quantification of surface runoff is important, especially for water resources management and hydrological research. However, the lack of in situ measurements of runoff in ungauged catchments or poorly gauged catchments is a major problem when quantifying runoff spatially. In general, rainfall-runoff models are used to estimate runoff at ungauged basins, usually with derived model parameterisation from gauged catchments with hydrologically similar characteristics (Blöschl, 2005). However, spatiotemporal heterogeneity of climate and catchment characteristics, as well as anthropogenic impacts, may cause uncertainties in predicting runoff from rainfall-runoff models. Therefore, the International Association of Hydrological Sciences (IAHS) initiated a program aiming to predict or forecast realistic estimates of ungauged basins, also known as Predictions in Ungauged Basins (PUB) by quantifying uncertainties in the predictions (Sivapalan et al., 2003).

Using models to predict runoff generation in ungauged basins remains challenging. Therefore, the adoption of nearby observations in such studies may be better as their inherent properties provide better estimates than numerical modelling. In terms of methodology, geostatistical interpolation could be a viable method as observations can be perfectly matched. In geostatistical kriging, weights of the nearest observations to an unknown location are calculated by minimising the variances; hence the weighted average of nearby observations is used to estimate a location without observations. However, exploitation of geostatistical kriging in streamflow hydrology is uncommon (Skøien \& Blöschl, 2007). Whereas variables such as rainfall can be measured at a point, this is not possible for streamflow because each streamflow measurement corresponds to its upstream area. That is, streamflow measurements have a large "support" (Skøien \& Blöschl, 2007) dictated by topography and drainage. Skøien et al. (2006) developed a geostatistical interpolation method considering river network topology named as top-kriging to estimate 100 year flood events at ungauged catchments; the method was primarily based on the work of Gottschalk (1993) and Sauquet et al. (2000) but with improvements. More recently, Skøien et al. (2014) implemented the top-kriging approach developed in Skøien et al. (2006) into an R package called rtop. The rtop package offers topological kriging technique for observations with irregular supports, such as different catchment areas. Moreover, runoff estimates along river networks at ungauged basins can be easily derived from rtop. However, one key disadvantage of rtop is that it cannot account for the spatial variations in rainfall, which is the primary covariate of runoff generation.

Here we address this issue and produce a reliable gridded runoff dataset across Victoria, with the broader aim of advancing our understanding of landscape water balance dynamics in future studies. The method section of this paper explains how rainfall was incorporated into the statistical model of runoff generation as an external drift, the residual interpolation in rtop and post-hoc adjustments to form the final estimated gridded runoff dataset. The later section discusses the results and the reliability of the method and the dataset.

\subsection{Plain summary of Skøien et al. (2006) work}

As mentioned above, interpolating runoff over space is more complicated than the interpolation of precipitation or evapotranspiration as topography governs the accumulation of runoff over space. Therefore, Skøien et al., (2006) developed top kriging to estimate discharge at ungauged catchments by accounting for both catchment and river network connectivity along with adopting flow distances rather than employing Cartesian distances between observations as in normal kriging. The distribution of top kriging weight can be explained by two categories: interpolation of weights (Figure 1.a) and extrapolation of weights (Figure 1.b).

In the case of interpolation (Figure 1.a), let's consider three gauges at the same distance $\left(x_{1}=x_{2}\right)$ from the point to be interpolated. Top kriging prioritises hydrological connectivity; therefore, it assigns zero weight to adjacent hydrologically disconnected gauges. Among the above and below hydrologically connected two catchments, the weight distribution is not equal (as in ordinary kriging) because top kriging takes into account the overlap area with ungauged catchments when assigning kriging weights. A catchment with a larger overlapping area is assigned a higher kriging weight than a smaller overlapping area.

In the case of extrapolation (Figure 1.b), let's consider one catchment is hydrologically connected and the other is not but with the same distance to the ungauged catchment. For this type of situation, top kriging assigns a lower weight to hydrologically disconnected catchments considering the similarity between adjacent catchments, whereas hydrologically connected catchments receive a higher weight. However, if the hydrologically connected gauged catchment is far away from the ungauged catchment, but the hydrologically unconnected catchment is closer, the top kriging tends to assign a higher weight to the adjacent catchment.

These situations get complicated when it comes to real river networks; however, the above theories apply accordingly. The weights are obtained by regularised variograms and top kriging theory can be found in Skøien et al., (2006) and Skøien et al. (2014). 


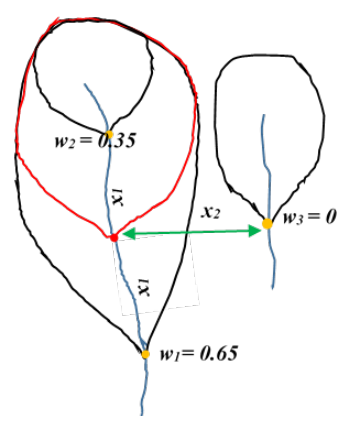

a) Interpolation

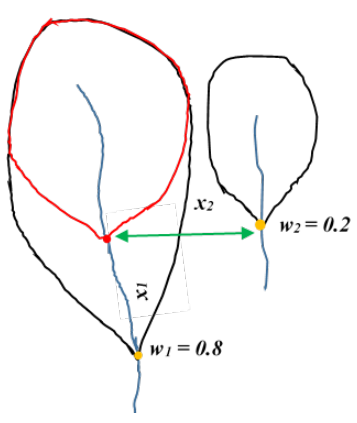

b) Extrapolation

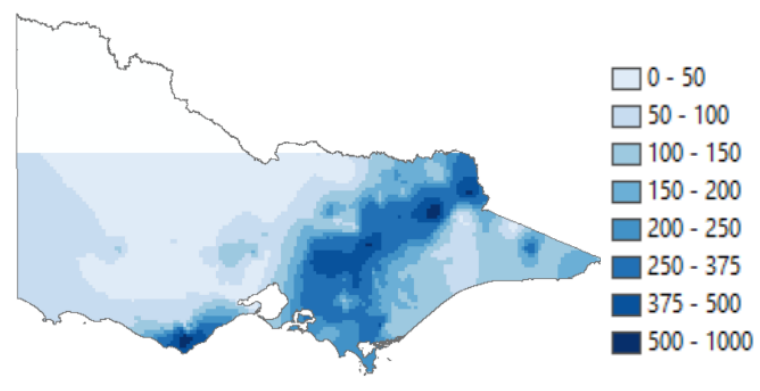

Figure 2. Average annual interpolation runoff $(\mathrm{mm})$ by adopting Skøien et al. (2014) work.

Figure 1. Schematic diagram to explain distribution of kriging
weights in the case of a) Interpolation and b) Extrapolation (The catchments with black coloured boundaries represent gauged catchments, and catchments with red coloured boundaries represent the ungauged catchments). Note, arbitrary weights are assigned for visualising purposes.

As a beginning to this study, we adopted rtop package in Skøien et al. (2014) to directly interpolate raw catchment runoff over Victoria during 1982 -2012 (Figure 2) to later compare results with our improved method which incorporates rainfall as an external drift to runoff generation.

\section{DATA AND METHOD}

\subsection{Data}

Discharge data aggregated to water years (March-February) from 154 unimpaired catchments and their area boundaries used in Saft et al. (2021) were adopted for runoff interpolation across Victoria. In general, higher runoff can be observed in the East part of Victoria as that region receives higher annual rainfall around 1500 $\mathrm{mm} / \mathrm{year}$ on average, whereas comparatively lower runoff can be observed in the West region with lower annual rainfall below $1000 \mathrm{~mm} /$ year (Fowler et al., 2020). Highlands are prominent in the East of Victoria, whereas lands are flatter in the West region. Annual average catchment runoff and annual average rainfall data (AWAP rainfall from the BoM website) are shown in Figure 3. Note that the semiarid north-west area of Victoria has not been included due to it being semiarid with negligible surface runoff.

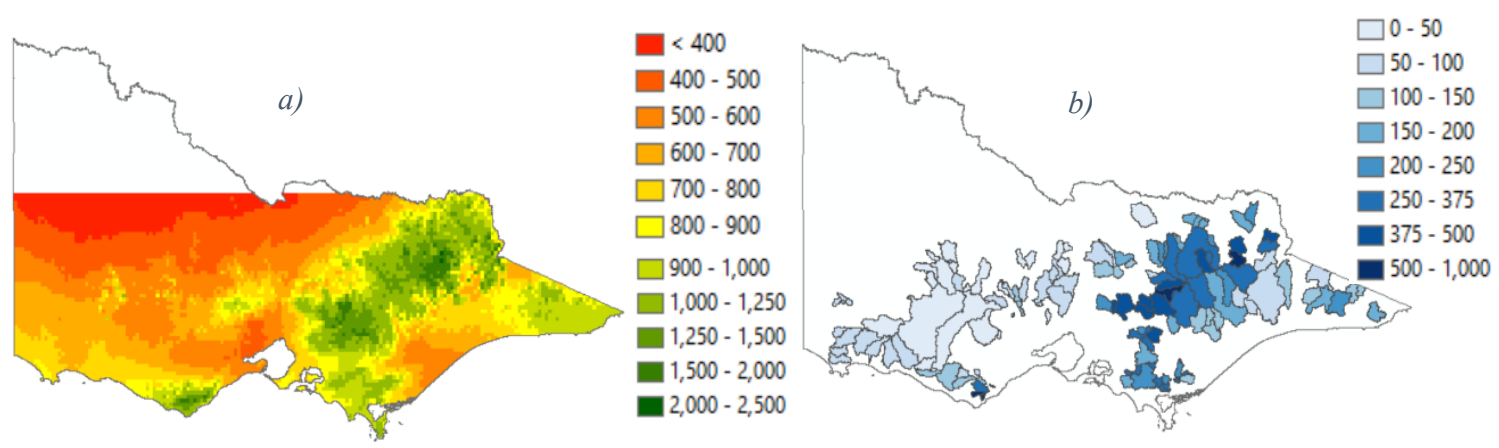

Figure 3. a) Annual average AWAP rainfall (mm), b) Annual average runoff (mm) of 154 catchments in Victoria.

\subsection{Method}

\subsubsection{Derivation of catchment runoff residuals}

A key disadvantage of the existing top kriging method is that it is unable to integrate information about the spatial distribution of rainfall, which is the main covariate of runoff generation. Moreover, the integration of rainfall into the method is advantageous as it indirectly accounts for variations in topography across the study area. Therefore, to overcome this limitation, we adopted a two-stage process where annual streamflow at each grid cell is first predicted using a simple relationship with annual rainfall, then the interpolation process interpolates the residuals rather than the raw flow values.

In initial testing, an exponential function was trialed between annual rainfall and annual runoff. However, this was problematic as it predicted runoff ratio values exceeding 1.0 for higher rainfall values. Thus, we moved to 
a system where the function predicts annual runoff ratio directly (Figure 4) and the functional form ensures values less than 1.0. The Richards equation (Richards, 1959) (note, this is a different Richards to the author well known in soil hydraulics) is a sigmoidal function (also known as "S-curve") employed to predict runoff ratio (and hence runoff) at grid scale. Optimised parameters for the Richards equation were obtained by minimising the sum of absolute error between observed catchment annual runoff and aggregated predicted gridded runoff at the 154 catchments considering all years during 1982-2012. Eq.1 shows the fitted Richards equation for runoff ratio and the subsequent prediction of gridded runoff can be expressed by multiplying the gridded runoff ratio with gridded AWAP rainfall.

$$
\frac{Q}{P}=\frac{1}{\left(1+10^{0.00046 *(-2886.6-P)}\right)^{111.9}}
$$

where, $Q / P$ - Gridded runoff ratio; $P$ - Gridded rainfall (i.e.: AWAP rainfall); $Q$ - Predicted gridded runoff. The fitted Richards function applied on the grid-scale and the scatter plot between annual predicted and observed runoff at catchment scale are shown in Figure 4 and Figure 5, respectively. Figure 5 shows modest bias at high runoff; however, the subsequent method ensures that the observed streamflow is preserved because the residuals of this equation are interpolated and then added back to the raw output of the equation, returning the original value.

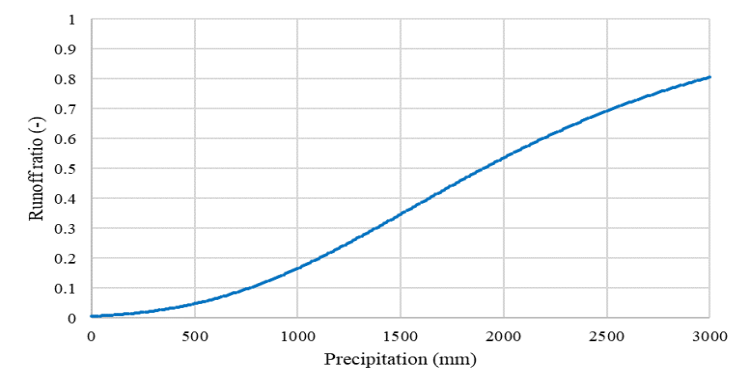

- fit line

Figure 4. The model applying to grid scale.

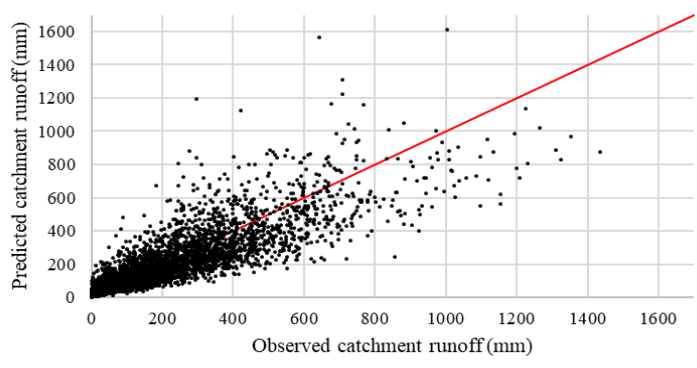

- $1: 1$ line

Figure 5. Scatter plot between model predicted annual gridded runoff aggregated over catchments and observed annual runoff during $1982-2012$.

For each year separately, the residuals between observed runoff and grid aggregated predicted runoff for every catchment were interpolated over Victoria. Although 154 catchments were employed in this study, the catchments with missing observations in any year were removed from the dataset before yearly interpolation of residuals in rtop. The example code in Skøien et al. (2014) was adopted to this study with adjustments. The main difference between Skøien et al. (2014) and this study is that Skøien et al. (2014) requires catchment shapefiles for each ungauged catchment to be estimated, whereas this study replaced the ungauged catchment shapefiles with gridded shapefiles to cover Victoria to obtain gridded outputs.

To derive top kriging weights, rtop provides the optimised point variogram (Figure 6, in black) and regularised semivariograms (Figure 6, categorised into different area bins) for catchment residuals in each year (Figure 6 shows the results for an example water year, 1987). Then, cross validations were performed to check the relative performance of the top-kriging model for each year. Finally, the interpolated gridded residuals were added back to the gridded predicted runoff to obtain adjusted gridded runoff.

\subsubsection{Post-hoc adjustments to adjusted gridded runoff}

Initial application of the above method was problematic because the gridded runoff dataset showed negative values of runoff for some grid cells. This situation arises when strongly negative residuals are co-located with low values of runoff from Eq. 1. One possible option would be to simply replace the negative values with zero, but it was felt the final result would be unrealistic. Therefore, a smoothing model was adopted from Kavetski \& Kuczera (2007) to correct negative values of runoff to non-zero values (Eq.2 \& Figure 7). This equation applies across the full range of real values (i.e. it shifts positive values too) but these shifts are negligible for most positive real values.

$$
\begin{gathered}
Q_{\text {neg corec pred,grid }}=k * m_{M} *\left(\bar{T}_{M}+\ln \left(1+\exp \left(-\bar{T}_{M}\right)\right)\right) \\
\bar{T}_{M}=\frac{\left(T-T_{0}\right)}{m_{M}}
\end{gathered}
$$


where, $\mathrm{k}$ - slope of the expected line; $m_{M}=10$ in Figure 7, an arbitrary value to the adjustment which was decided by trials to minimise the deviations of runoff values around zero (See Figure 7), $T_{0}$ - expected y value at 0 ;

After the post-hoc adjustment on gridded runoff, a regional specific case was found to be unrealistic to their actual runoff ratios (Black grid cluster in East region in Figure 10.a in the Results section). It was found that rain shadow occurs in that area because of the adjacent alpine region which resulted in higher interpolated residuals and led to negative gridded runoff. To avoid this problem, a proxy boundary was created around the rain shadowed area by introducing a pseudo catchment to the dataset with zero residual in each year; hence, the residual interpolation was repeated for each year.

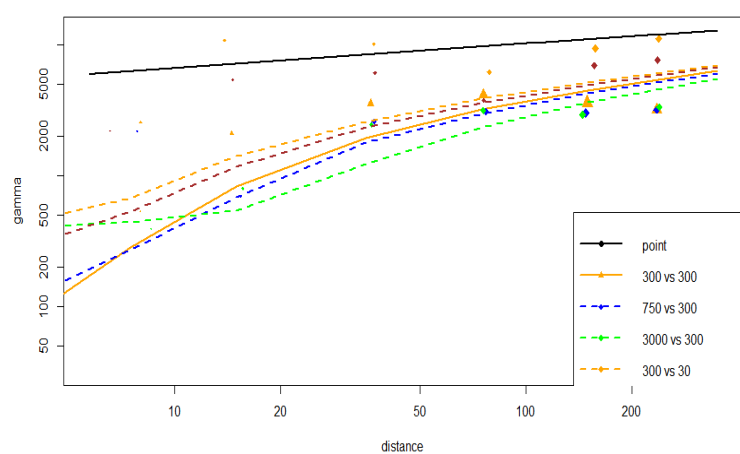

Figure 6. Optimised point variogram (in black) and regularised semivariograms for different area bins.

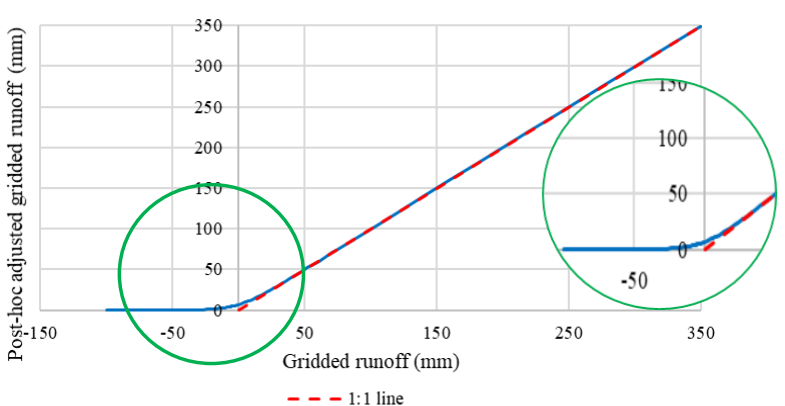

Figure 7. Post-hoc adjustment model adopted from Kavetski \& Kuczera, (2007).

\section{RESULTS \& DISCUSSION}

\subsection{Results of method}

By adopting the above methodology, the gridded runoff dataset was developed over Victoria during 1982 2012 period. Some of the following results show only one year, and similar results were obtained for other years. The catchment residuals derived for the year 1987 are shown in Figure 8.a, while Figure 8.b presents interpolated gridded residuals over Victoria.
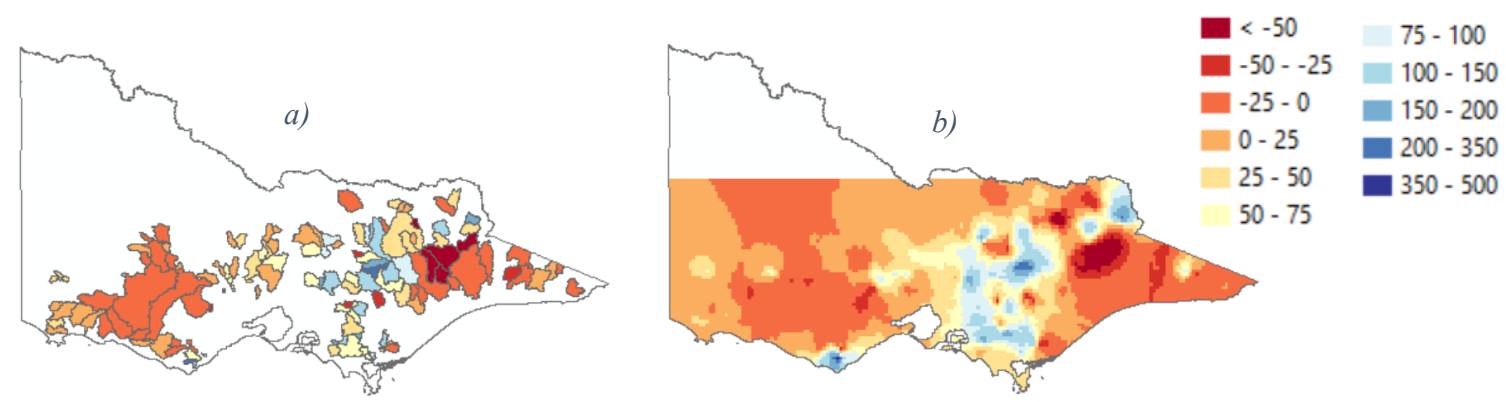

Figure 8. a) Distribution of runoff residuals ( $\mathrm{mm}$ ) between observed and area average predicted runoff over Victoria in 1987. b) Map of interpolated gridded residuals (mm) using rtop package in 1987.

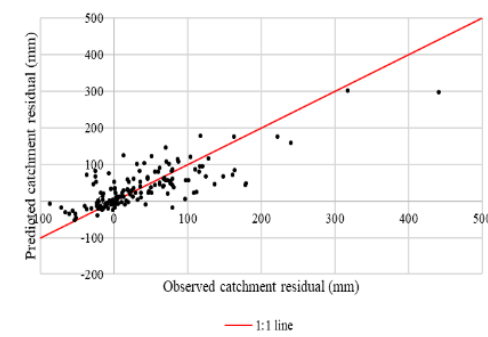

a) $\quad$ RMSE $=43.4 \mathrm{~mm}$

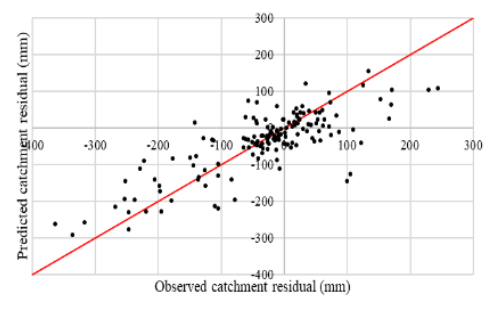

- $1: 1$ line

b) RMSE $=62.8 \mathrm{~mm}$

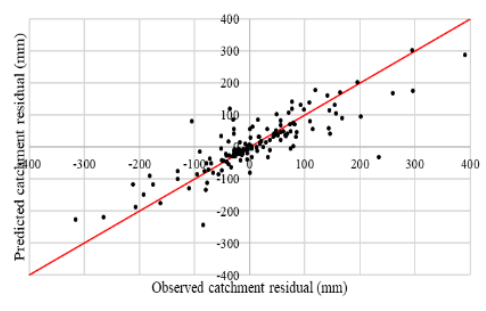

- $1: 1$ line

c) RMSE $=51.3 \mathrm{~mm}$

Figure 9. Scatter plots between catchment residual predictions from rtop cross validation and catchment observed residuals in year a) 1987 b) 1992 c) 2000. 
Cross validation based statistics for example years 1987, 1992, and 2000 present $43.4 \mathrm{~mm}, 62.8 \mathrm{~mm}$, and 51.3 $\mathrm{mm}$ of root mean square error (RMSE) of residuals respectively, which depict quite a higher error in interpolated residuals. In general, the absolute value of residuals tended to be slightly underestimated, which is typical in kriging cross validation. However, the coefficient of determination $\left(\mathrm{R}^{2}\right)$ values were $0.63,0.64$, and 0.72 which are in the range of model fitting scores in Skøien et al. (2014). Their study interpolated the raw flow values, whereas our focus on residuals is expected to make prediction more difficult due to the removal of the stabilizing influence of the underlying rainfall field. Thus, it is pleasing that performance is still comparable.

As mentioned in the method, some of the grids resulted in negative runoff values when negative residuals are strongly co-located with low values of predicted runoff by the Richards equation as shown in Figure 10.a.

\subsection{Post-hoc adjustments and final results}

The post-hoc adjustment has been done to correct negative values from Eq.2, and the resultant map is shown in Figure 10.b for the year 1987. As mentioned in the method, the negative corrected gridded runoff predictions in the rain shadow area still seem to be unrealistic as they show annual runoff below $10 \mathrm{~mm}$ and thus runoff ratios of less than 0.01 . However, this area is expected to generate more runoff than $10 \mathrm{~mm}$ as it receives annual rainfall of around $600 \mathrm{~mm}-650 \mathrm{~mm}$ on average. Therefore, the residual interpolation and subsequent steps had to be repeated after introducing a pseudo catchment as mentioned above in section 2.2.3.

The distribution of observed and predicted catchment runoff datasets from 1982 - 2012 are shown in Figure 11. The predicted dataset matches satisfactorily with the observed dataset. Moreover, the predicted and observed catchment runoff for the whole period in Figure 12 reconfirms that the method works as expected (RMSE of $19.9 \mathrm{~mm} / \mathrm{yr}$ and $\mathrm{R}^{2}$ of 0.99 ). However, the match is not exact because these fields are projected onto the grid cells and each cell is given the average value, which degrades the information content of the original field. When a catchment contains only a portion of a grid cell, the contribution calculated for that grid cell will be different to the contribution that would have been calculated prior to the degradation. Nonetheless, the above mentioned results indicate that the method performs satisfactorily in individual years.
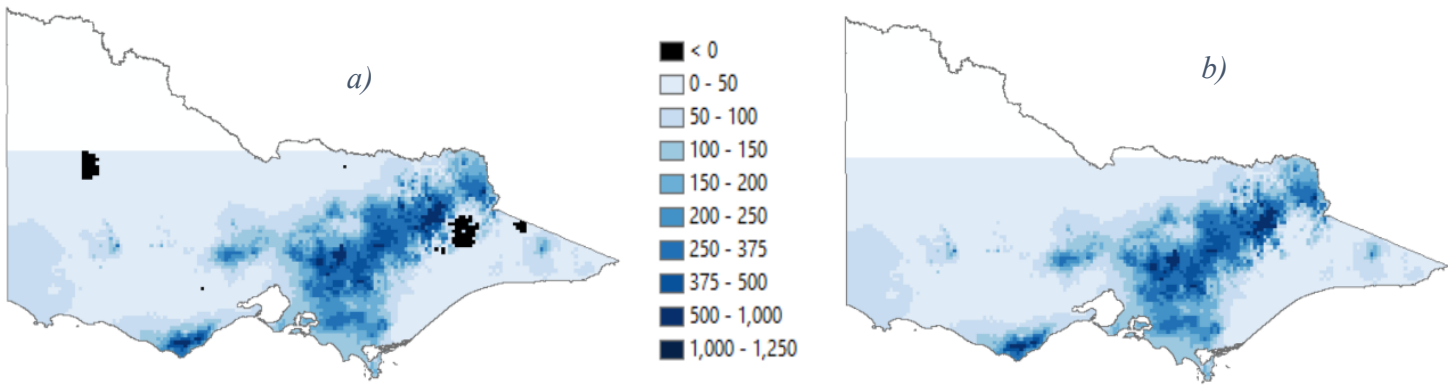

Figure 10. Predicted gridded runoff (mm) a) after adjusted by interpolated gridded residuals in 1987 (Grids with negative values are presented in black), b) after negative value correction in 1987 using Eq.2.

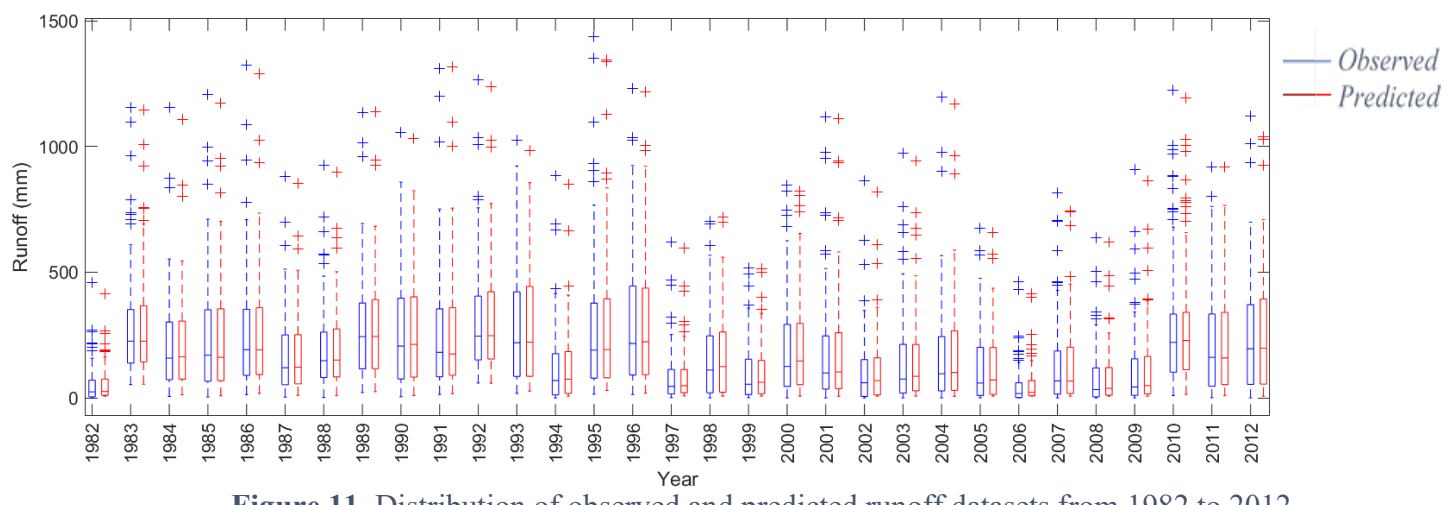

Figure 11. Distribution of observed and predicted runoff datasets from 1982 to 2012.

Finally, the average annual gridded runoff in Victoria during 1982-2012 is shown in Figure 13. When comparing the runoff estimates by incorporating rainfall as an external drift (Figure 13) with runoff estimates from Skøien et al. (2014) method (Figure 2), Figure 2 results seem to be averaged over the space, resulting in underestimated runoff in the higher values than the method we developed while an improved spatial variability of runoff can be seen in Figure 13 as it accounts for the spatial variability of rainfall. 


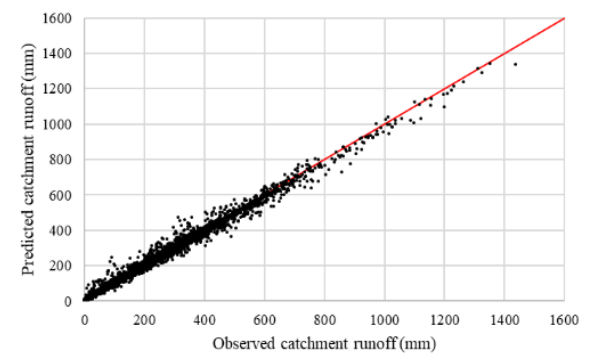

Figure 12. Predicted catchment runoff vs observed catchment runoff over 1982-2012.

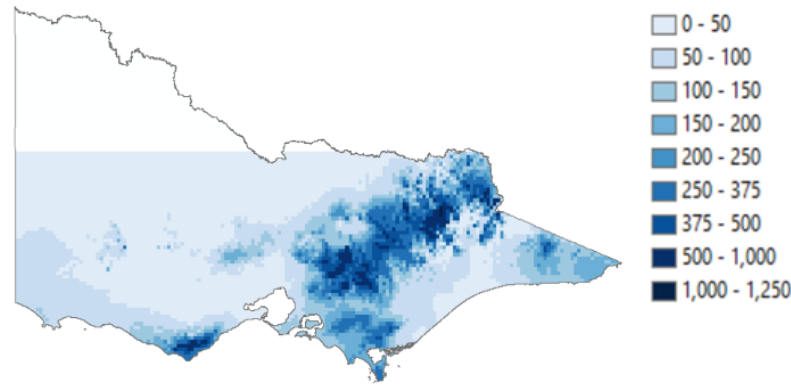

Figure 13. Annual average predicted gridded runoff $(\mathrm{mm})$ in Victoria (1982-2012)

\section{CONCLUSION}

The paper presents a new gridded runoff dataset over Victoria covering $1982-2012$. The developed dataset can contribute toward advancing hydrological research in Victoria as it presents a spatiotemporally continuous reliable runoff dataset. This paper also provides an updated methodology, applicable anywhere around the world, which allows interpolated runoff estimates to better reflect known spatial patterns in rainfall, which is not possible with the standard methodology (rtop R package; Skøien et al., (2014)). Overall, this paper presents an improved top-kriging methodology and an interpolated runoff dataset for most of Victoria to the hydrologic research community. The dataset is publicly available at https://doi.org/10.5281/zenodo.5454798.

\section{ACKNOWLEDGEMENTS}

This research is funded by an Australian Research Council Linkage Project (LP180100796), with partner organisations Victorian Department of Environment, Land, Water and Planning, and Melbourne Water.

\section{REFERENCES}

Blöschl, G., 2005. Rainfall-Runoff Modeling of Ungauged Catchments. Encycl. Hydrol. Sci. https://doi.org/10.1002/0470848944.hsa140

Fowler, K., Knoben, W., Peel, M., Peterson, T., Ryu, D., Saft, M., Seo, K.W., Western, A., 2020. Many Commonly Used Rainfall-Runoff Models Lack Long, Slow Dynamics: Implications for Runoff Projections. Water Resour. Res. 56. https://doi.org/10.1029/2019WR025286

Gottschalk, L., 1993. Interpolation of runoff applying objective methods. Stoch. Hydrol. Hydraul. 7, 269-281. https://doi.org/10.1007/BF01581615

Kavetski, D., Kuczera, G., 2007. Model smoothing strategies to remove microscale discontinuities and spurious secondary optima im objective functions in hydrological calibration. Water Resour. Res. 43, 1-9. https://doi.org/10.1029/2006WR005195

Richards, F.J., 1959. A flexible growth function for empirical use. J. Exp. Bot. 10, 290-301. https://doi.org/10.1093/jxb/10.2.290

Saft, M., Peel, M., Peterson, T., 2021. Explaining hydrological shift and non-recovery after prolonged drought. Nat. Geosci. (in Rev.

Sivapalan, M., Takeuchi, K., Franks, S.W., Gupta, V.K., Karambiri, H., Lakshmi, V., Liang, X., McDonnell, J.J., Mendiondo, E.M., O'Connell, P.E., Oki, T., Pomeroy, J.W., Schertzer, D., Uhlenbrook, S., Zehe, E., 2003. IAHS Decade on Predictions in Ungauged Basins (PUB), 2003-2012: Shaping an exciting future for the hydrological sciences. Hydrol. Sci. J. 48, 857-880. https://doi.org/10.1623/hysj.48.6.857.51421

Skøien, J.O., Blöschl, G., 2007. Spatiotemporal topological kriging of runoff time series. Water Resour. Res. 43, 1-21. https://doi.org/10.1029/2006WR005760

Skøien, J.O., Blöschl, G., Laaha, G., Pebesma, E., Parajka, J., Viglione, A., 2014. Rtop: An R package for interpolation of data with a variable spatial support, with an example from river networks. Comput. Geosci. 67, 180-190. https://doi.org/10.1016/j.cageo.2014.02.009

Skøien, J.O., Merz, R., Blöschl, G., 2006. Top-kriging - Geostatistics on stream networks. Hydrol. Earth Syst. Sci. 10, 277-287. https://doi.org/10.5194/hess-10-277-2006 